\title{
Time-Lapse STM Studies of Diastereomeric Cinchona Alkaloids on Platinum Metals
}

\author{
Markus Wahl, ${ }^{*, \dagger}$ Matthias von Arx, ${ }^{\dagger, \ddagger}$ Thomas A. Jung, ${ }^{\dagger, \S}$ and Alfons Baiker ${ }^{\ddagger}$ \\ Institut für Physik der Universität Basel, Klingelbergstrasse 82, CH-4056 Basel, Switzerland, Labor für Mikro- \\ und Nanotechnologie, Paul Scherrer Institut, CH-5232 Villigen, Switzerland, and Departement für Chemie und \\ Angewandte Biowissenschaften, ETH Zürich, Hönggerberg, HCI, CH-8093 Zürich, Switzerland
}

Received: May 11, 2006; In Final Form: July 24, 2006

\begin{abstract}
The adsorption of cinchonidine (CD) and cinchonine $(\mathrm{CN})$ on $\mathrm{Pt}(111)$ and $\mathrm{Pd}(111)$ single crystals has been investigated by means of scanning tunneling microscopy (STM) in an ultrahigh vacuum system. In timelapse series the mobilities of different adsorption species have been determined on a single molecule basis and with varying hydrogen background pressures in the system. The diastereomeric cinchona alkaloids, CD and $\mathrm{CN}$, which are widely used as chiral modifiers of platinum group metals in catalytic enantioselective hydrogenation, showed similar adsorption modes and diffusion behavior on $\operatorname{Pt}(111)$, except that the flatly adsorbed $\mathrm{CN}$ molecules which were free (not in a dimer/cluster) were significantly more mobile than their $\mathrm{CD}$ analogues. CD adsorbed on $\mathrm{Pd}(111)$ showed similar adsorption modes as observed on $\mathrm{Pt}(111)$ but at considerably higher mobility of the flatly absorbed species already in the low-pressure region. The observed adsorption behaviors are discussed in the context of independent ATR-IR measurements and theoretical calculations. Special emphasis is put on the nonlinear effect observed in hydrogenation reactions with $\mathrm{CD} /$ $\mathrm{CN}$ mixtures. Our observations corroborate that this effect is mainly a consequence of the different adsorption strengths of $\mathrm{CD}$ and $\mathrm{CN}$ on $\mathrm{Pt}$.
\end{abstract}

\section{Introduction}

Since the first observation of individual molecules on a welldefined metal surface by STM in $1987 / 88,1,2$ a wealth of data have been gained on a big variety of molecules on various metal surfaces. In recent years, several groups focused on molecule surface combinations related to heterogeneous catalysis ${ }^{3-5}$ or even enantioselective heterogeneous catalysis. ${ }^{6-8}$ In the latter case, STM investigations are of special interest, as the arrangement of the chiral molecules on the catalytic surface (chiral site/ extended chiral domain), which is crucial for the efficiency of the catalyst system, can be imaged in real space. In an ideal case, such investigations could reveal the dynamic interplay between chiral site and reactant on the surface on a single molecule level. However, for the time being-and probably the near future-this remains a vision.

One of the difficulties of such STM investigations lies in the intrinsic reactivity of catalytically active surfaces. In a textbook example, Raval and co-workers showed that the behavior of tartaric acid, which is used as chiral modifier in the enantioselective hydrogenation of $\beta$-ketoesters, shows a completely different behavior on the nonreactive $\mathrm{Cu}$ and the reactive $\mathrm{Ni}$ surfaces, respectively. ${ }^{7,9}$ The strong interaction of tartaric acid with the Ni surface affects the structure of the molecules and/ or molecular clusters on the surface significantly. The platinumcinchona-alkaloid system is another well-known catalyst system for heterogeneous enantioselective hydrogenation reactions. ${ }^{10}$ In a recent publication we reported on our first STM results obtained with the chiral modifier cinchonidine (CD) on the Pt(111) surface under the influence of coadsorbed hydrogen, which

* Author to whom correspondence should be addressed. E-mail: markus.wahl@unibas.ch.

†nstitut für Physik der Universität Basel.

$\doteqdot$ ETH Zürich.

$\S$ Paul Scherrer Institut.
CHART 1: Molecules Investigated in This Work: Cinchonidine (CD) and Its Diastereomer Cinchonine $(\mathrm{CN})$<smiles></smiles><smiles>N#CN1C[C@H]2CC[C@H]1CN2[C@H](O)c1ccnc2ccccc12</smiles>

\begin{tabular}{ll|l} 
& $\mathrm{CD}$ & $\mathrm{CN}$ \\
\cline { 2 - 3 } $\mathbf{3}$ & $\mathrm{R}$ & $\mathrm{R}$ \\
$\mathbf{4}$ & $\mathrm{S}$ & $\mathrm{S}$ \\
$\mathbf{8}$ & $\mathrm{S}$ & $\mathrm{R}$ \\
$\mathbf{9}$ & $\mathrm{R}$ & $\mathrm{S}$
\end{tabular}

is present in hydrogenation reactions. ${ }^{11}$ We could identify two different adsorption modes of CD: (i) due to the significantly different appearance in the STM images and (ii) due to the increased mobility of only one of these surface species in the presence of a standing hydrogen pressure.

Intrigued by this peculiar behavior we now extended our studies in two ways: first we included cinchonine $(\mathrm{CN})-\mathrm{a}$ diastereomer of $\mathrm{CD}$ - and second the other catalytically relevant surface $\operatorname{Pd}(111)$ into our investigations (Chart 1). The aim of this extension was to identify similarities and differences between the two modifiers on both surfaces with special emphasis on their diffusion behavior. An interpretation of the results in view of the known catalytic behavior and the existing 
knowledge gained by other surface analytical methods and ab initio calculations is given.

From the literature a number of direct measurements of single adsorbate diffusion are known.,5,12-14 Most of these studies focus on adatoms or small molecules, where diffusion can be described as a result of uncorrelated displacements or hops between adjacent minima of the corrugated potential energy hypersurface, representing the adsorbate-substrate interaction. ${ }^{15}$ The frequency with which those hops are observed is the socalled hopping rate $h$. This lattice gas model is appealing because it is simple and many of its assumptions have been found to agree well with the direct experimental observation. However, for larger organic molecules, similar studies on the surface mobility are rare ${ }^{16}$ and very often restricted to (110) oriented surfaces. This excellent experimental simplification reduces the diffusion process to a $1 \mathrm{D}$ problem and typically does not allow for rotational degrees of freedom. Furthermore, the energy dissipation to the substrate is rather weak for large organic molecules and therefore the assumption of uncorrelated hops is too simplistic: once a molecule has reached the energy of the diffusion barrier, it does not stop in the adjacent energy minimum but has a high probability to move on for several minima. By including these so-called long jumps, the lattice gas model has been slightly modified. ${ }^{17}$

However, for the more complex adsorbate-substrate system $(\mathrm{CD} / \mathrm{CN}$ on $\mathrm{Pt}(111))$ treated in this paper it is very difficult to gain information on the precise adsorption site, which involves several surface atoms. Together with the structural flexibility of $\mathrm{CD}$ and $\mathrm{CN}$ this yields a very complex difficult-to-determine potential energy hypersurface for the diffusion process. Furthermore, the complexity is enhanced by structural changes, which are likely to occur in the molecule and the surface in the course of the adsorption process. ${ }^{18}$ As a consequence the simple lattice gas model will not be able to describe the diffusion process adequately for our system. Nevertheless, to be able to compare the adsorption and diffusion behavior of the different surface species of $\mathrm{CD}$ and $\mathrm{CN}$ on both metal surfaces we had to define a method to quantify the surface mobility. As a measure for the surface mobility we therefore determined the average displacement of all surface species populations independently at four to five different hydrogen background pressures.

\section{Experimental Methods}

All experiments were performed in a multichamber UHV system that allows the preparation and characterization of molecular layers (down to $0.1 \mathrm{ML}$ ) on atomically clean substrates. $\mathrm{Pt}(111)$ and $\mathrm{Pd}(111)$ single crystals were cleaned by repeated $\mathrm{Ar}$ ion sputtering $\left(800 \mathrm{eV}, 4 \times 10^{-7} \mathrm{mbar}\right)$ and subsequent annealing to $1000 \mathrm{~K}$ via electron impact. Annealing, at times, in the presence of a standing oxygen pressure of $5 \times$ $10^{-8}$ mbar removed any organic impurities and led to atomically clean surfaces. Due to the high sticking coefficient of both Pd and especially $\mathrm{Pt}$, special care had to be taken to make sure that the base pressure in the system remained in the $10^{-11} \mathrm{mbar}$ range and STM measurements have not been delayed more than 2-3 $\mathrm{h}$ once the samples were prepared. A separate chamber was used to deposit the $\mathrm{CD}$ and $\mathrm{CN}$ molecules by sublimation from a resistively heated tantalum crucible at temperatures $\leq 200$ ${ }^{\circ} \mathrm{C}$. With the mass spectrometer attached to the deposition chamber no $\mathrm{CD} / \mathrm{CN}$ mass fragments could be detected during the sublimation process. The substrate was kept at room temperature during the deposition process and a cooling trap attached to the chamber was cooled with liquid nitrogen to keep residual gas pressure at a minimum. A custom-built quartz microbalance was used to measure the deposition rate.

All samples were characterized with a home-built STM operating at room temperature. All STM images and time-lapse imaging sequences were obtained in constant-current mode by recording the vertical tip movement of an etched and electron bombarded tungsten tip. The standing hydrogen pressure (pressure range: $10^{-10}$ to $10^{-6}$ mbar) in the STM chamber was controlled in situ by a leak valve.

The average displacement data shown were obtained from a time-lapse series of STM images, where scan range and scan speed were set appropriately in order to be able to distinguish the different appearance and mobility of the molecules while still imaging a statistically meaningful number of molecules in one frame. In some cases this was a difficult task, because good topographical imaging of individual molecules is typically dependent on the tip condition, which can vary during experiments and at the different scan speeds used. High-resolution images have been achieved at slow scan speeds $(\approx 200 \mathrm{~nm} / \mathrm{s})$, while higher scan speeds $(\approx 600 \mathrm{~nm} / \mathrm{s})$ were required to track very mobile molecules.

The image sequences were evaluated by tracking 10 to 15 single molecules by using the particle analysis tool in the Nanotec WSXM STM data processing software. The resulting $x y$-pathes were drift corrected and their integral displacement was calculated by summing up the distances measured from frame to frame, fully neglecting the fact that due to the limited scan speed the molecules may have moved a longer distance between the images. Therefore all mobilities given here are lower limits. By taking the slopes of such integral displacements and averaging over the whole population of a surface species, we obtained the average displacement and its standard deviation in nanometers per minute.

\section{Results}

CD on $\operatorname{Pt}(111)$. Compared to the results of our earlier study, ${ }^{11}$ the refined analysis method described above allows a more detailed description of the surface diffusion of the different surface species populations. In the new series of STM sequences (81.5 s/frame), the molecules were tracked individually while the standing hydrogen pressure in the STM chamber was increased stepwise from $1 \times 10^{-10}$ to $2 \times 10^{-6} \mathrm{mbar}$ and the mobility of the molecules was determined for each surface species population and for each hydrogen pressure.

In general, the new results support the conclusions made earlier: two clearly distinct adsorption modes can be identified. They have been assigned as flat (quinoline ring system parallel to the surface, strong interaction with the surface, low mobility) and tilted adsorption modes (quinoline ring system inclined to the metal surface, weaker interaction with the surface, high mobility at elevated hydrogen pressure), respectively, as discussed in detail in our previous work. ${ }^{11}$

On the other hand, the new data set revealed more details in the diffusion behavior. In particular, a difference in the mobility of the flatly adsorbed molecules-depending on their local surface density-becomes apparent. This surface species population has to be divided into two groups. The molecules which are isolated (no other molecules in close proximity, filled triangles in Figure 1b) show a slight increase in mobility when increasing the hydrogen pressure, while the molecules arranged in a dimer/cluster (one or more molecules in close vicinity, filled squares in Figure 1b) stay practically immobile on the time scale and in the pressure range of our experiments. The slight mobility increase of the isolated molecules mainly originates from a 

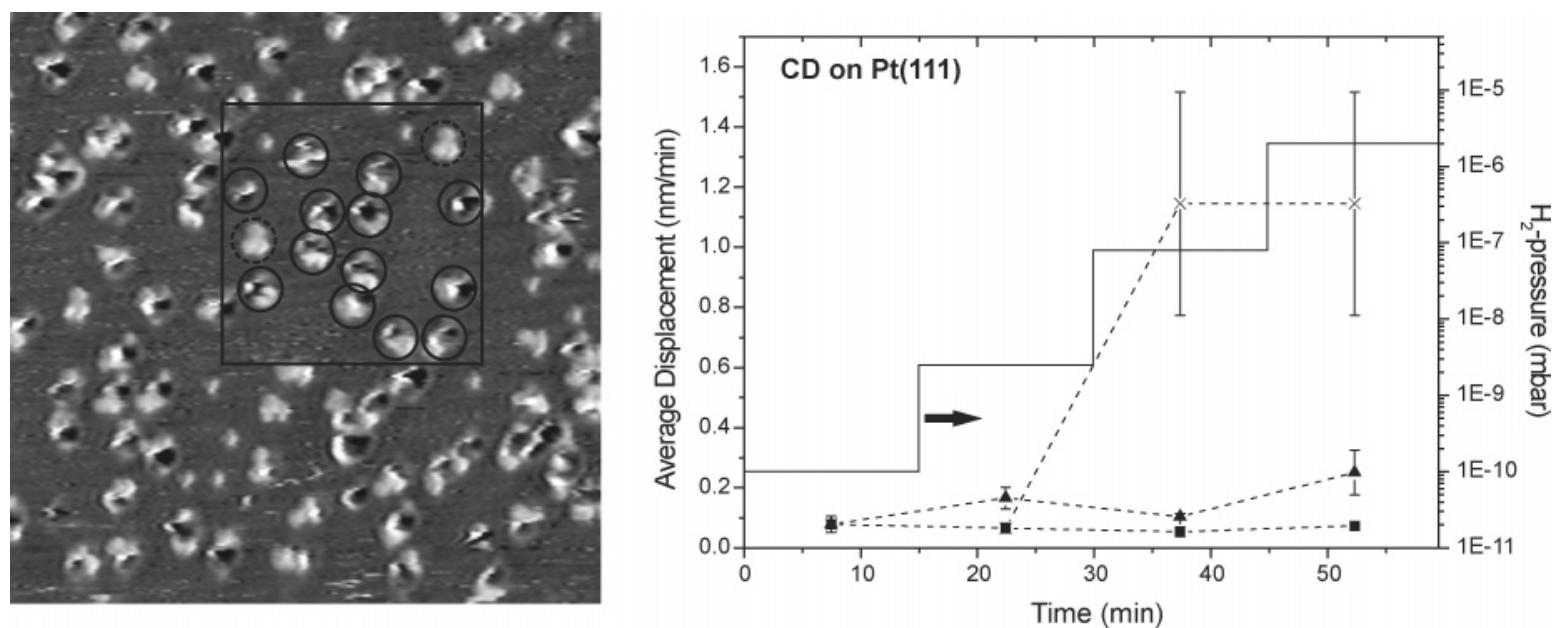

Figure 1. (a) STM image of CD $(0.27 \mathrm{ML})$ on $\mathrm{Pt}(111)$ at low hydrogen pressure $\left(2 \times 10^{-10} \mathrm{mbar}\right)$. Inside the marked area, representative for the whole sample, the two different adsorption modes of $\mathrm{CD}$ are highlighted. Circles designate the flatly adsorbed molecules, and dashed circles mark the molecules in the tilted adsorption mode. Image size $=25 \times 25 \mathrm{~nm}^{2} ; I=50 \mathrm{pA} ; U=1.0 \mathrm{~V}$. (b) Average displacement measured for CD on $\mathrm{Pt}(111)$. The step function represents the background hydrogen pressure during the time-lapse series. Filled symbols show the mobility of flatly adsorbed molecules, where the triangles stand for free molecules and the squares stand for molecules in clusters. Crosses represent molecules initially adsorbed in a tilted adsorption mode. They swap into a different adsorption mode with significantly increased mobility at elevated hydrogen pressures.
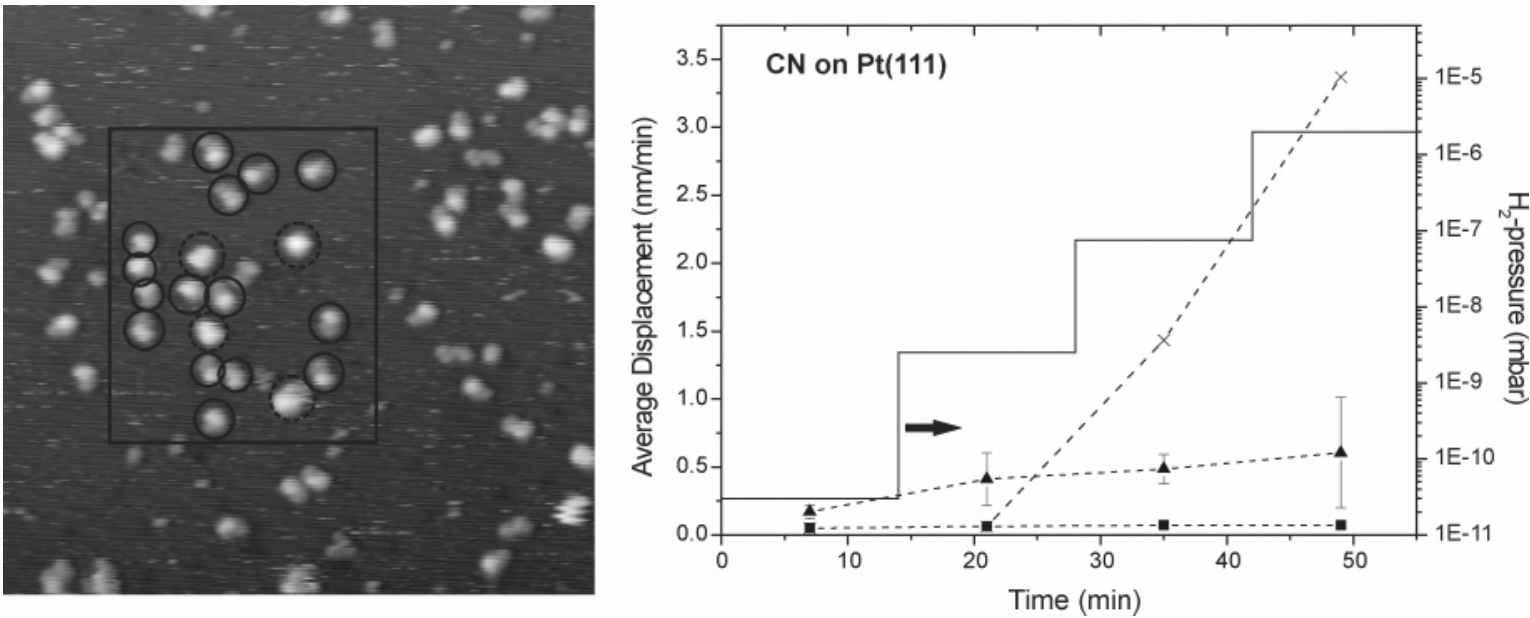

Figure 2. (a) STM image of $\mathrm{CN}(0.15 \mathrm{ML})$ on $\mathrm{Pt}(111)$ at a hydrogen pressure of less than $10^{-10} \mathrm{mbar}$. As for CD, the two different adsorption modes of $\mathrm{CN}$ are highlighted for all molecules inside the marked area. Circles designate the flatly adsorbed molecules. Dashed circles mark molecules in the tilted adsorption mode. Image size $=30 \times 30 \mathrm{~nm}^{2} ; I=25 \mathrm{pA} ; U=0.8 \mathrm{~V}$. (b) Average displacement measured for $\mathrm{CN}$ on $\mathrm{Pt}(111)$. The step function represents the background hydrogen pressure during the time-lapse series. Filled symbols show the mobility of flatly adsorbed molecules, where the triangles stand for free molecules and the squares stand for molecules in clusters. Crosses represent molecules initially adsorbed in a tilted adsorption mode. They swap into a different adsorption mode with significantly increased mobility at elevated hydrogen pressures.

wobbling motion of the molecules at elevated pressure: the molecules seem to be pinned to the surface by a part of the molecule while the remaining part of the molecule wobbles from side to side, typically in angles of $60^{\circ}$. The population of molecules with a tilted adsorption geometry (crosses in Figure 1b) show the same behavior as described earlier. ${ }^{11}$ They remain immobile until, at a threshold hydrogen pressure of $8 \times 10^{-8}$ mbar, the mobility increases instantaneously to a much higher value. At the same time the appearance of these molecules changes significantly. This transformation is irreversible as no reversal to the original appearance and mobility is observable upon lowering the hydrogen pressure back to the original value. The new set of data shows that the mobility increases by a factor of $\approx 10$ or even more in the course of this transformation. The average displacement value for this species has a relatively high variance (see error bars) because of the effect of clustering: On its track across the surface, such a molecule-at times-gets in close proximity to other molecules ("cluster"), which leads to a temporal decrease in the mobility. As the time during which an individual molecule remains inside such "clusters" varies from molecule to molecule, the average displacement value for the whole population varies. Of course this effect depends on the (local) coverage of the sample.

CN on Pt(111). CN differs from CD only in the absolute configuration of two of the four stereocenters (Chart 1). Therefore the two molecules are diastereomers. Because of the mirror plane symmetry of the stereocenters $\mathrm{C} 8$ and $\mathrm{C} 9$, which are crucial for the stereochemical control of the catalytic reaction, the two molecules are often called "near-enantiomers", An example of the topographic image obtained from $\mathrm{CN}$ on $\mathrm{Pt}(111)$ is given in Figure 2a. As observed for $\mathrm{CD}$, the $\mathrm{CN}$ molecules are distributed randomly on the surface, which indicates a low mobility due to a strong molecule-surface interaction. On first sight, the molecules appear quite different from the images obtained for CD (Figure 1a). This is mainly due to tip effects: from sample to sample, the very apex of an 

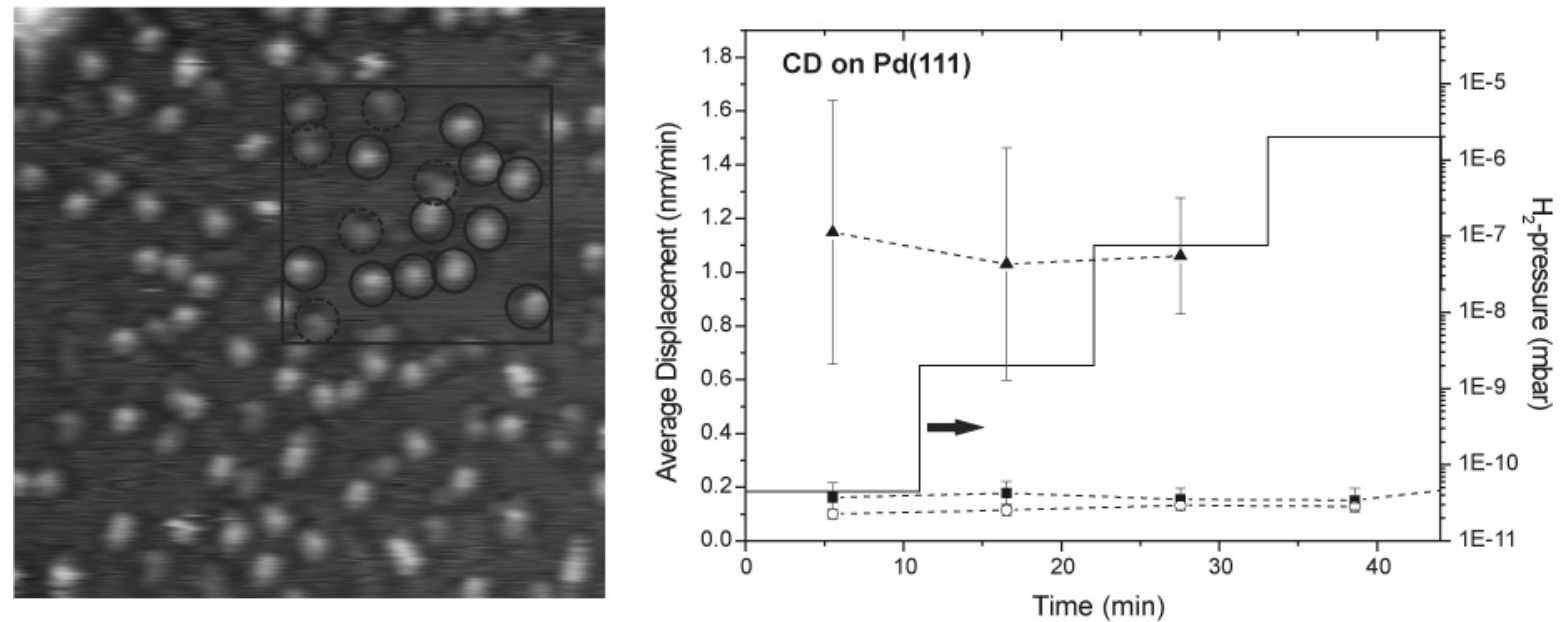

Figure 3. (a) STM image of CD (0.25 ML) on Pd(111) at low hydrogen pressure $\left(2 \times 10^{-10}\right.$ mbar). Inside the marked area, representative for the whole sample, two different adsorption modes of $\mathrm{CD}$ are highlighted. Circles designate the flatly adsorbed molecules, and dashed circles mark a different adsorption mode. Image size $=25 \times 25 \mathrm{~nm}^{2} ; I=22 \mathrm{pA} ; U=1.05 \mathrm{~V}$. (b) Average displacement measured for CD on Pd(111). The step function gives the background hydrogen pressure during the time-lapse series. Filled symbols show the mobility of flatly adsorbed molecules, where the triangles stand for free molecules and the squares stand for molecules in clusters. Outlined circles represent molecules adsorbed in a different adsorption mode, with a rather dark appearance.

STM tip changes, which leads to significant differences in the image of objects, in particular of isolated objects (in contrast to regular arrays of objects) such as $\mathrm{CD}$ or $\mathrm{CN}$, which are randomly distributed on the surface. Compared to the CD/Pt(111) sample shown in Figure 1a, the tip allowed only lower resolution in the case of the $\mathrm{CN} / \mathrm{Pt}(111)$ sample shown in Figure $2 \mathrm{a}$. As a result, the molecules appear a bit broader, and the characteristic up/down feature ${ }^{11}$ in the image of the flatly adsorbed CD molecules is not visible for the $\mathrm{CN}$ molecules in Figure 2a. Nonetheless, we assign the molecules appearing as a gray oval with a brighter spot, which are marked with a circle in Figure 2a, as flatly adsorbed molecules. This assignment is justified by the following facts: (i) with a lower resolution the flatly adsorbed molecules of CD appear exactly the same; (ii) by changing the scanning speed and/or the speed of the feedback regulation of the tip movement, the image of a flatly adsorbed $\mathrm{CD}$ in Figure 1a changes toward the appearance observed for the $\mathrm{CN}$ molecules in Figure $2 \mathrm{a}$ and vice versa; and (iii) the diffusion behavior of these molecules is very similar to the one observed for the flatly adsorbed CD molecules but clearly distinct from the molecules marked with dashed circles in Figure $2 a$.

The result of the analysis of the surface diffusion of $\mathrm{CN}$ on $\mathrm{Pt}(111)$ is given in Figure 2b. Again, the two surface species populations can be clearly discriminated concerning their mobility. The flatly adsorbed molecules in dimers or clusters are immobile while the ones which are free show a slight mobility, which is increasing by a factor of $\approx 2-3$ with increasing hydrogen pressure. In this respect, this surface species population differs from the corresponding surface species population of $\mathrm{CD}$ (compare filled triangles in Figures $1 \mathrm{~b}$ and 2b). The surface species population marked with dashed circles in Figure 2b shows the same behavior as the tilted CD molecules (compare crosses in Figures $1 \mathrm{~b}$ and $2 \mathrm{~b}$ ). At a hydrogen pressure of $7.5 \times 10^{-8} \mathrm{mbar}$, these molecules show an instantaneous increase in their mobility by more than a factor of 10 . For this reason we tentatively assign these molecules to a tilted adsorption geometry, in analogy to their CD counterparts. For the last two data points in Figure $2 \mathrm{~b}$ no error bars are given, because only a few molecules could be tracked. Due to their very high mobility, these molecules move out of view quickly, limiting the precision of the measurement of their average displacement.
Altogether this means that the diffusion behavior of $\mathrm{CD}$ and $\mathrm{CN}$ on $\mathrm{Pt}(111)$ in the presence and absence of hydrogen is very similar. Nevertheless, there is one significant difference: the flatly adsorbed CN molecules which are free (not in a dimer/ cluster) show a significantly higher mobility than their CD analogues.

CD on Pd(111). An STM image of $0.25 \mathrm{ML}$ of CD deposited onto a Pd(111) single crystal is shown in Figure 3a. Although this is a different substrate, again a random distribution of the molecules is observed and at least two different adsorption modes can be distinguished. One of them is clearly similar to the flat adsorption mode identified for $\mathrm{CD}$ and $\mathrm{CN}$ on Pt. Even though the resolution in the image shown is lower than that in the previous images, it is clearly visible that this adsorption mode consists of two parts: a rather flat, oval bottom part and a relatively flexible (under the influence of the tip) top part. This is in agreement with a flat adsorption mode as identified before. At the first sight of the time-lapsed image series we realized that already in the low pressure regime, the flatly adsorbed molecules, which are not part of a dimer/cluster, show a remarkably high mobility (filled triangles in Figure 3b). This is a significant difference from the mobility observed for the analogous surface species population on Pt (compare filled triangles in Figures $1 \mathrm{~b}$ and $3 \mathrm{~b}$ ). Since a large fraction of all molecules on Pd belong to this surface species population, the overall mobility in this image series is much higher than that on Pt.

The molecules marked with dashed circles in Figure 3a differ from the flatly adsorbed molecules in several ways. In the topographical image they lack the characteristic signature for the flexible top part, which is a clear sign for an adsorption geometry significantly different from the flat adsorption mode. However, the resolution obtained does not allow any conclusion on the precise adsorption geometry. The surface mobility of this surface species population (circles in Figure 3b) is clearly distinct from that of the flatly adsorbed molecules. There is virtually no mobility observable, independent of the local environment (free molecules vs dimers/clusters) and independent of the hydrogen pressure. This is a further justification for the identification of these molecules as a second surface species population. No flipping of molecules has ever been observed on $\operatorname{Pd}(111)$ for any surface species. 
Altogether this means that on $\operatorname{Pd}(111)$ the flatly adsorbed surface species population is the more mobile species, in contrast to the observations for $\mathrm{CD}$ and $\mathrm{CN}$ on $\mathrm{Pt}(111)$. In addition there seems to be no (measurable) effect of the hydrogen pressure on the surface diffusion on $\mathrm{Pd}$, at least in the pressure regime accessible in our experiments.

\section{Discussion}

It is interesting to relate the surface diffusion behavior of $\mathrm{CD}$ and $\mathrm{CN}$, and in particular the observed differences between them, to the well-known catalytic behavior of the Pt and Pd cinchona alkaloid system. As shown in Figures $1 b$ and $2 b$, the dynamic behavior of these two chiral modifiers on $\operatorname{Pt}(111)$ is very similar but not identical. This is not surprising, as one would expect an identical behavior only for real enantiomers, while $\mathrm{CD}$ and $\mathrm{CN}$ are "near-enantiomers" or to be precise, diastereomers. This is also reflected in a wealth of catalysis experiments, which show that $\mathrm{CD}$ and $\mathrm{CN}$ behave almost like enantiomers, yielding the products with different absolute configuration at the newly formed stereocenter. However, the efficiency of the reaction concerning the enantiodifferentiation is slightly different for CD and CN. Typically the enantiomeric excess (ee) to the (R)-product achieved with $\mathrm{CD}$ is higher (by a few percent) than the one with $\mathrm{CN}$ to the (S)-product, as already observed in the original studies by Orito et al. ${ }^{19}$ The origin for this small difference is still not clear. No direct evidence for a difference in the adsorption mode or the conformational behavior has been found so far. The first indirect clue has been given by Huck et al., ${ }^{20}$ who found that predominantly the product with the absolute configuration expected for a reaction with $C D$ as chiral modifier was formed, when using 1:1 mixtures of $\mathrm{CD}$ and $\mathrm{CN}$ or even an excess of CN. However, from these experiments it could not be concluded whether the origin of this nonlinear effect lies in a difference of the adsorption strength of the two modifiers and/or (subtle) differences in the conformational or geometrical configuration between modifier and reactant, responsible for the enantiodifferentiation.

Our STM and diffusion analysis data provide further important evidence for answering this longstanding question. In principle, either different surface species populations with different chiral efficiencies or differences in the adsorption strength could be at the origin of the nonlinear effect observed. While different surface species population cannot be ruled out with the presently available experimental evidence, our STM and diffusion analysis data clearly indicate a difference in the adsorption strength between the flatly adsorbed $\mathrm{CD}$ and $\mathrm{CN}$ molecules as the origin of the nonlinear effect. Indeed this is the only difference we observe between the two modifiers in our diffusion analysis. Compared to $\mathrm{CD}$, the average displacement is higher for the flatly adsorbed $\mathrm{CN}$ molecules. As the adsorption of these molecules is through strong chemisorption, including several chemical bonds between several surface atoms and various carbon atoms of the quinoline ring system, ${ }^{18,21}$ the average displacement is a direct measure of the adsorption strength. As a consequence the flatly adsorbed $\mathrm{CN}$ molecules adsorb less strongly on the Pt surface than the flatly adsorbed CD molecules. As all existing mechanistic models consider the flatly adsorbed $\mathrm{CD} / \mathrm{CN}$ molecules as the catalytically relevant species, ${ }^{10}$ our results could explain the nonlinear effect: If CD and $\mathrm{CN}$ are coadsorbed, the concentration of the flatly adsorbed $\mathrm{CD}$ molecules is higher on the surface than the concentration of the analogous surface species of $\mathrm{CN}$. Of course this effect could be complemented by subtle differences in the geometrical configuration of $\mathrm{CD} / \mathrm{CN}$ and reactant on the surface, which are beyond the resolution power attainable by STM for this system.

The other interesting observation is the difference in the diffusion behavior of $\mathrm{CD}$ on $\mathrm{Pt}(111)$ and $\mathrm{Pd}(111)$. While on $\mathrm{Pt}$ the population of the flatly adsorbed molecules is almost immobile (Figure 1b), the flatly adsorbed CD molecules show a high mobility on Pd. This indicates that the adsorption strength for this surface species of CD is much higher on Pt than on Pd. Again this is in line with a well-known fact: to obtain good enantioselectivities in hydrogenation reactions over $\mathrm{Pd}$ much higher $\mathrm{CD}$ concentrations are needed than on $\mathrm{Pt}^{22,23}$ Due to the competition with reactant and solvent molecules a high concentration of CD is needed to cover a significant amount of the surface with CD molecules. On Pt, however, the interaction of $\mathrm{CD}$ with the surface is so strong that small amounts of CD are enough to compete with other potential adsorbates. As a result, too high $\mathrm{CD}$ concentrations lead to a drastic decrease in reaction rate on $\mathrm{Pt}$, since the reactants have to compete with $\mathrm{CD}$ for the adsorption sites.

Finally the differences between $\mathrm{Pt}$ and $\mathrm{Pd}$ observed here complement existing knowledge attained by ATR-IR spectroscopy, ${ }^{24}$ where it was found that tilted adsorption geometries are more abundant on Pd than on Pt. This is clearly supported by our STM measurements. Our mobility analysis shows that on $\mathrm{Pd}$ the surface species population with the unknown adsorption geometry is less mobile than the flatly adsorbed molecules. Obviously there are adsorption geometries that bind more strongly to the Pd surface than the flat adsorption mode of CD. As a result, the flat adsorption mode is less abundant on Pd than on Pt, as observed in ATR-IR spectroscopy.

\section{Conclusions}

Our analysis of the appearance and surface mobility of CD and $\mathrm{CN}$ on platinum metals by time-lapse STM imaging has revealed some interesting differences between the different surface species populations. The interpretation of these findings sheds some new light on the possible reasons for the nonlinear effects found in the enantioselective hydrogenation over platinum metals modified by cinchona alkaloids. Therefore our work shows that STM investigations can complement other surface analytical methods and quantum chemical calculations in order to gain insight into the mechanistic aspects of this complex catalyst system.

Acknowledgment. We thank Nanonis Inc. for the fruitful collaboration on the data aquisition. Financial support from the Swiss National Science Foundation is gratefully acknowledged.

Supporting Information Available: Figure showing a sequence of example frames taken from an STM movie of CD deposited on $\mathrm{Pt}(111)$. This material is available free of charge via the Internet at http://pubs.acs.org.

\section{References and Notes} 267.

(1) Gimzewski, J. K.; Stoll, E.; Schlittler, R. R. Surf. Sci. 1987, 181,

(2) Ohtani, H.; Wilson, R. J.; Chiang, S.; Mate, C. M. Phys. Rev. Lett. 1988, 60, 2398.

(3) Somorjai, G. A. Appl. Surf. Sci. 1997, 121, 1.

(4) Zambelli, T.; Trost, J.; Wintterlin, J.; Ertl, G. Phys. Rev. Lett. 1996 76,795

(5) Tansel, T.; Magnussen, O. M. Phys. Rev. Lett. 2006, 96, 026101.

(6) Lopinski, G. P.; Moffatt, D. J.; Wayner, D. D.; Wolkow, R. A. Nature 1998, 392, 909.

(7) Lorenzo, M. O.; Baddeley, C. J.; Muryn, C.; Raval, R. Nature 2000 , 404, 376. 
(8) Bonello, J. M.; Williams, F. J.; Santra, A. K.; Lambert, R. M. J. Phys. Chem. B 2000, 104, 9696. 130 .

(9) Humblot, V.; Haq, S.; Muryn, C.; Raval, R. J. Catal. 2004, 228,

(10) Burgi, T.; Baiker, A. Acc. Chem. Res. 2004, 37, 909.

(11) von Arx, M.; Wahl, M.; Jung, T. A.; Baiker, A. Phys. Chem. Chem. Phys. 2005, 7, 273

(12) Mo, Y. W. Phys. Rev. Lett. 1993, 71, 2923.

(13) Linderoth, T. R.; Horch, S.; Laegsgaard, E.; Stensgaard, I.; Besenbacher, F. Phys. Rev. Lett. 1997, 78, 4978.

(14) Schaub, R.; Wahlstrom, E.; Ronnau, A.; Laegsgaard, E.; Stensgaard, I.; Besenbacher, F. Science 2003, 299, 377.

(15) Gomer, R. Rep. Prog. Phys. 1990, 53, 917.
(16) Schunack, M.; Linderoth, T. R.; Rosei, F.; Laegsgaard, E.; Stensgaard, I.; Besenbacher, F. Phys. Rev. Lett. 2002, 88, 156102.

(17) Dobbs, K. D.; Doren, D. J. J. Chem. Phys. 1992, 97, 3722

(18) Vargas, A.; Ferri, D.; Baiker, A. J. Catal. 2005, 236, 1.

(19) Orito, Y.; Imai, S.; Niwa, S. Nippon Kagaku Kaishi 1980, 4, 670.

(20) Huck, W. R.; Burgi, T.; Mallat, T.; Baiker, A. J. Catal. 2003, 216 , 276.

(21) Vargas, A.; Baiker, A. J. Catal. 2006, 239, 220.

(22) Huck, W. R.; Mallat, T.; Baiker, A. New J. Chem. 2002, 26, 6.

(23) Nitta, Y.; Watanabe, J.; Okuyama, T.; Sugimura, T. J. Catal. 2005 236,164 .

(24) Ferri, D.; Burgi, T.; Baiker, A. J. Catal. 2002, 210, 160. 